\title{
Cytidine deaminase activity as a measure of acute inflammation in rheumatoid arthritis
}

\author{
P W THOMPSON , ${ }^{1}$ D D JONES ${ }^{2}$ AND H L F CURREY ${ }^{1}$
}

From the 'Bone and Joint Research Unit, The London Hospital Medical College, London E1 2AD; and the ${ }^{2}$ Department of Biochemistry, Bronglais General Hospital, Aberystwyth SY23 1ER, Wales

SUmmary Cytidine deaminase (CD), a cytoplasmic enzyme, is thought to leak out of damaged cells and can be measured in fluids by a simple biochemical assay. This study has shown that serum CD activity is raised in rheumatoid arthritis (RA) compared with osteoarthritis (OA). Synovial fluid (SF) CD activity was always less than the corresponding serum activity (mean $\mathrm{SF} /$ serum ratio $=0.6$ ) in $\mathrm{OA}$ but up to 22 times greater than the corresponding serum activity in RA (mean SF/serum ratio=13.1), suggesting CD production in inflammatory joints. Evidence to support the $S F$ neutrophil as a cell of $C D$ origin is provided by the $C D$ gradient running from cells to $\mathrm{SF}$ to synovium. The close correlation between SF CD activity and neutrophil count $(r=0.93)$ indicates that SF CD activity is an accurate measure of acute synovial inflammation. Weak correlation of serum $C D$ activity with erythrocyte sedimentation rate $(E S R)(r=0.44)$ and $C$ reactive protein (CRP) $(r=0.49)$ implies that $C D$ estimations supply different though related information about rheumatoid disease activity. We suggest that $\mathrm{CD}$ released from damaged neutrophils diffuses from all inflamed joints into the blood, so that serum CD activity may provide an integrated measure of joint inflammation more specific than traditional measures such as the ESR.

Key words: cytidine aminohydrolase, nucleoside deaminases, nucleoside aminohydrolases, joint inflammation.

Cytidine deaminase (CD) is a cytoplasmic enzyme that catalyses the hydrolytic deamination of cytidine, deoxycytidine, and their analogues.

$$
\text { cytidine }+\mathrm{H}_{2} \mathrm{O} \stackrel{\mathrm{CD}}{\longrightarrow} \text { uridine }+\mathrm{NH}_{3}
$$

Although its exact physiological role is uncertain, it may provide a salvage pathway for pyrimidine nucleosides produced during the breakdown of nucleic acids. ${ }^{1}$

Clinical interest in the enzyme developed when some patients with acute myeloblastic leukaemia proved resistant to treatment with cytosine arabinoside, an antileukaemic drug known to be degraded by $C D .^{2}$ The finding that their marrow cells had

Accepted for publication 4 June 1985.

Correspondence to $\mathrm{Dr}$ Paul W Thompson, Bone and Joint Research Unit, The London Hospital Medical College, London E1 2AD. raised levels of $C D$ led to the suggestion that $C D$ inhibition might be used to augment treatment. ${ }^{3}$

A simple assay has been developed ${ }^{14}$ and the distribution of $C D$ in human tissues determined (Fig. 1). ${ }^{1}$

In a similar manner to the release of creatine phosphokinase from cardiac muscle during myocardial infarction $C D$ is thought to leak out of damaged cells and can be measured quantitatively in serum. Serum CD levels are raised in a variety of conditions, including rheumatoid arthritis (RA) (Table 1). ${ }^{1}$

The relationship between serum $C D$ levels and the development of pre-eclamptic toxaemia is now well established, though the organ of origin of $C D$ is uncertain. Estimation of maternal serum $C D$ activity has been recommended as a screening test and is in routine use at several centres. ${ }^{4}$

This paper reports the results of a study designed to investigate the site of origin and significance of $\mathrm{CD}$ activity in RA patients. 


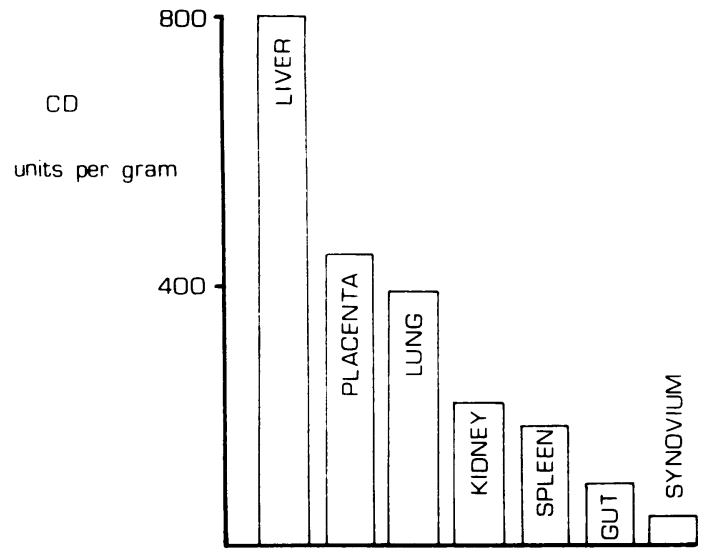

\begin{tabular}{|c|c|c|}
\hline CELLS & CD units per $10^{6}$ cells & SD \\
\hline NEUTROPHILS & 14.7 & 0.73 \\
LYMPHOCYTES & 0.73 & 0.07 \\
RBC & NONE & - \\
\hline
\end{tabular}

Fig. 1 Distribution of $C D$ in human tissues. Results are the means of 10 postmortem samples. Heart, gall bladder, pancreas, muscle, bladder, and bone contained no $C D$ activity.' (RBC=red blood cells).

\section{Materials and methods}

\section{LON D O N}

Samples of blood, synovial fluid (SF), and synovium were collected from patients with osteoarthritis (OA) and definite or classical RA during routine venepuncture, joint aspiration, or joint replacement at The London Hospital.

Blood was taken into ethylenediaminetetra-acetic acid (EDTA) or plain glass tubes. SF samples collected without anticoagulant were centrifuged at

Table 1 Serum $C D$ activity in disease

\begin{tabular}{ll}
\hline Raised $C D$ activity & Normal $C D$ activity \\
\hline Septacaemia & Influenza \\
Pyelonephritis & Cystitis \\
Cellulitis & Polymyositis \\
Pneumonia & Obstructive jaundice \\
Ulcerative colitis & Pancreatitis \\
Rheumatoid arthritis & Arteritis \\
Pre-eclampsia & Essential hypertension of pregnancy \\
Intrauterine death & \\
Viral hepatitis & \\
Liver metastases & \\
Osteomyelitis & \\
\hline
\end{tabular}

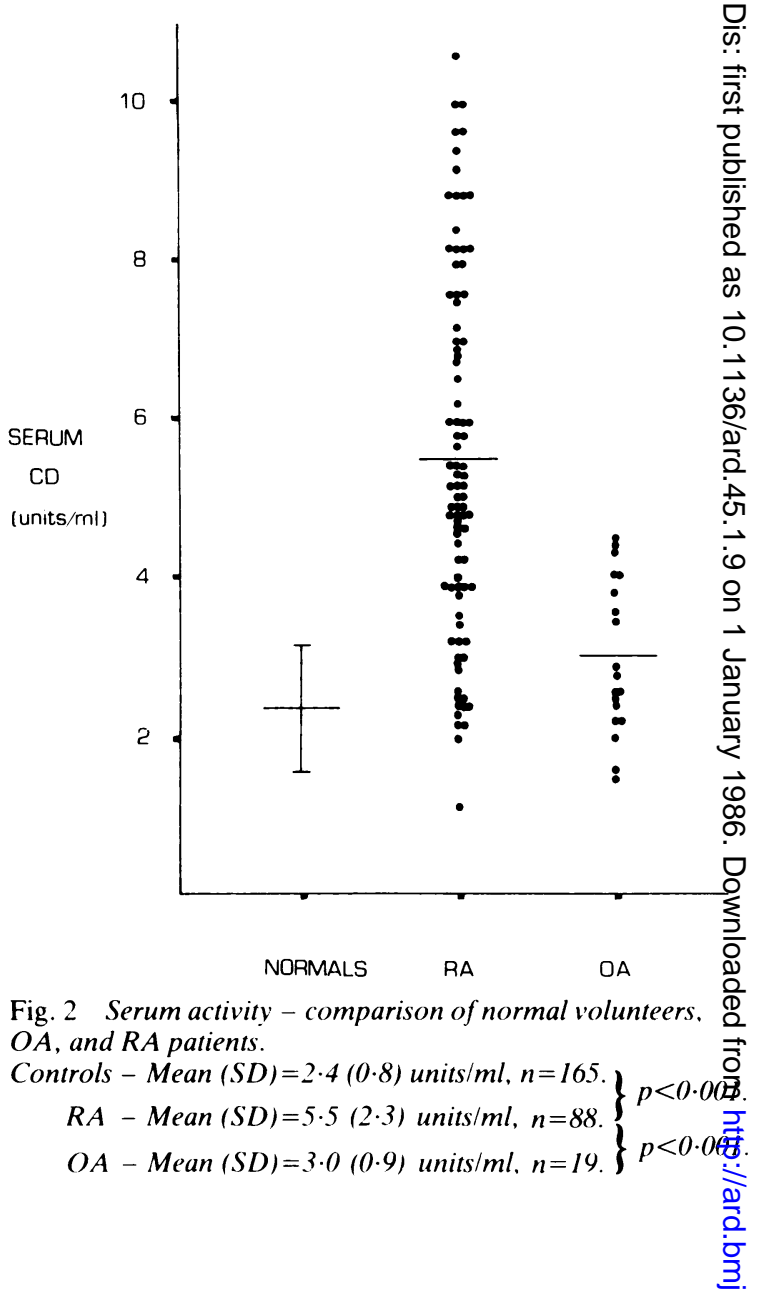

$1000 \mathrm{~g}$ for 10 minutes and supernatant separated. In $\stackrel{\delta}{\delta}$ some cases a recorded volume of SF was centrifuged and the pellet which adhered to the bottom of the 의 tubes separated from the supernatant.

Loss of serum enzyme activity has been shown to be minimal if serum is frozen within four hours, ${ }^{1}$ but $\vec{N}$ in order to test if the same were true for $\mathrm{SF}$ a number of samples were stored at $22^{\circ} \mathrm{C}$ and aliquots ${ }^{N}$ taken at intervals, centrifuged, and the supernatant $\underset{\omega}{N}$ collected.

All samples were stored at $-20^{\circ} \mathrm{C}$ within four hours of collection. Samples were coded, packed in $\frac{\varnothing}{\varnothing}$ solid carbon dioxide, and sent from London to $\stackrel{\mathscr{?}}{+}$ Aberystwyth once per fortnight. In all cases samples 70 arrived still frozen within 24 hours of departure.

Synovial fluid leucocyte counts were performed with a haemocytometer (Hawksley BS 748) on $\overrightarrow{\mathbb{Q}}$ samples collected into EDTA, and differential cell counts performed on stained smears of EDTA SF by 
a single observer without knowledge of the fluid CD activity.

Estimations of the erythrocyte sedimentation rate (ESR) were made by the modified Westergren method.

\section{A B E R Y S T W Y T H}

Samples were thawed and processed immediately without knowledge of the patients' diagnosis or condition.

Synovium and cellular pellets were homogenised with distilled water in a tissue grinder. The extract was ultrasonicated for 10 minutes at $8 \mathrm{kHz}$ and then centrifuged to remove debris. ${ }^{1}$

Estimations of CD activity were carried out with cytidine as a substrate, and the ammonia liberated was measured by a modified Berthelot reaction without protein precipitation. Details of this technique have been published and show high inter- and intrabatch reproducibility (coefficient of variation $1.6 \%$ and $2 \cdot 8 \%$ respectively). ${ }^{1}$

Enzyme activity is expressed as the amount of ammonia produced per unit time. One unit being equivalent to the release of $10^{-4} \mu \mathrm{mol}$ ammonia per minute. For fluids the activity is expressed as units $/ \mathrm{ml}$, for tissues as units/g wet weight, and for cells as units $/ 10^{6}$ cells.

The $\mathrm{CD}$ activity in the cellular component of a unit volume of SF was calculated by the following equation:

$\mathrm{CD}$ activity in the cells from $1 \mathrm{ml} \mathrm{SF}=\frac{\text { total } \mathrm{CD} \text { of } \mathrm{SF} \text { cell sample }}{\text { volume of } \mathrm{SF} \text { in } \mathrm{ml}}$

In an attempt to exclude enzyme release from liver or kidney estimations of $\gamma$-glutamyltransferase $(\gamma-G T)$ and creatinine were made on the serum samples.

C-reactive protein (CRP) was measured by the turbidometric method with a Hyland laser nephelometer, creatinine by the Technicon AA11 continuous flow system using alkaline picrate, and $\gamma$-GT by the calorimetric method as supplied by Boehringer.

Analysis of data employed the techniques of linear correlation and Student's $t$ test.

\section{Results}

\section{S E R U M}

There was a large variation in serum $C D$ activity among the 88 RA patients tested (Fig. 2). The mean CD activity $(5.5$ units $/ \mathrm{ml})$ was significantly higher than in both OA patients $(3.0$ units $/ \mathrm{ml})$ and a reference population of Welsh students ${ }^{1}(2 \cdot 4$ units/ml) $(\mathrm{p}<0 \cdot 001)$.

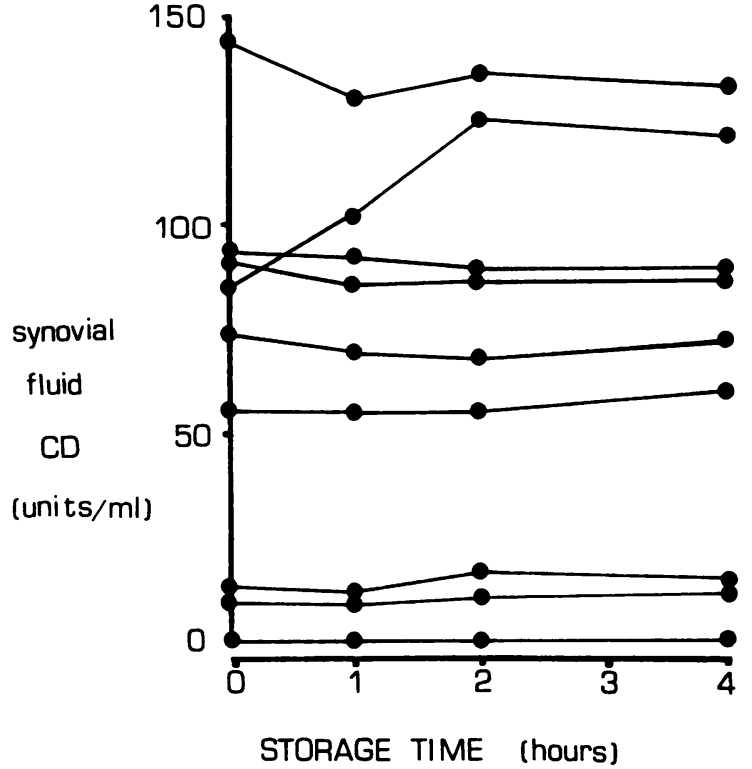

Fig. $3 C D$ activity during short term storage of synovial fluid at $22^{\circ} \mathrm{C}$.

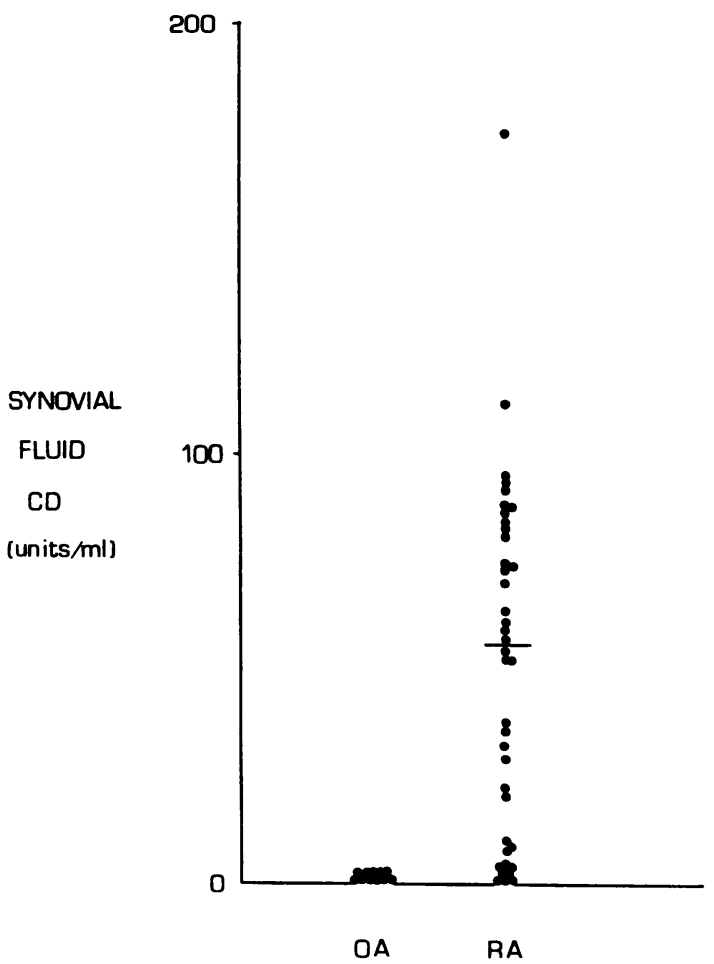

Fig. 4 Synovial fluid $C D$ activity in $O A$ and $R A$ patients.

$O A-$ Mean $(S D)=1 \cdot 8(0 \cdot 8)$ units $/ \mathrm{ml}$.

$R A-$ Mean $(S D)=52.3(40.0)$ units $/ \mathrm{ml}.\} \quad p<0.001$. 
SYNOVIAL FLUID

From Fig. 3 it can be seen that short term storage had minimal effect on SF CD activity.

SF CD activity was markedly raised in RA patients compared with OA patients (Fig. 4) and correlated well with SF neutrophil count $(r=0.93)$ (Fig. 5).

The CD activity of the cellular component of a unit volume of RA SF (mean $=89$ units $/ \mathrm{ml}$ ) was greater than the corresponding supernatant $C D$ activity (mean $=53$ units $/ \mathrm{ml})(\mathrm{p}<0.05)$, and the cellular and supernatant $\mathrm{CD}$ levels correlated well $(\mathrm{r}=0.86)$ (Fig. 6).

\section{SYNOVIAL FLUID/SERUM RATIO}

SF CD activity was lower than the corresponding serum activity for the OA patients (mean SF/serum ratio $=0 \cdot 6, n=11$ ) but up to 22 times higher for the RA patients (mean SF/serum ratio $=13 \cdot 1, n=16$ ). Fig. 7 shows weak positive correlation between serum and SF CD levels in RA patients $(r=0.45)$.

\section{SYNOVIUM}

For the nine samples of rheumatoid synovium that were processed the mean (SD) CD activity was 53 (48) units/g and the mean (SD) CD activity of the corresponding SF was 25 (34) units/ml.

COMPARISON WITH ESR AND CRP

Comparison of serum CD activity with ESR and CRP was made in 49 cases of RA. In one case the serum $\gamma$-GT was raised at $86 \mathrm{IU} / \mathrm{l}$ but in all other cases $\gamma$-GT and creatinine measurements fell within

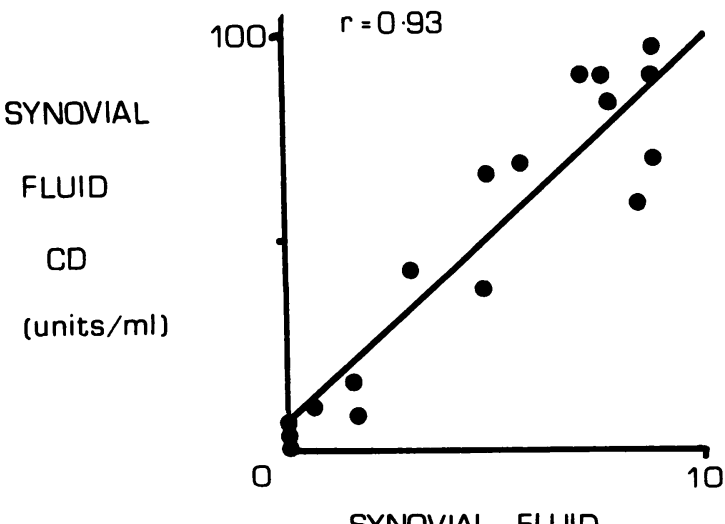

NEUTROPHILS

(cells $\times 10^{9 / 1}$ )

Fig. 5 RA synovial fluid scattergram. $C D$ versus neutrophil count. $r=0.93, n=17$.

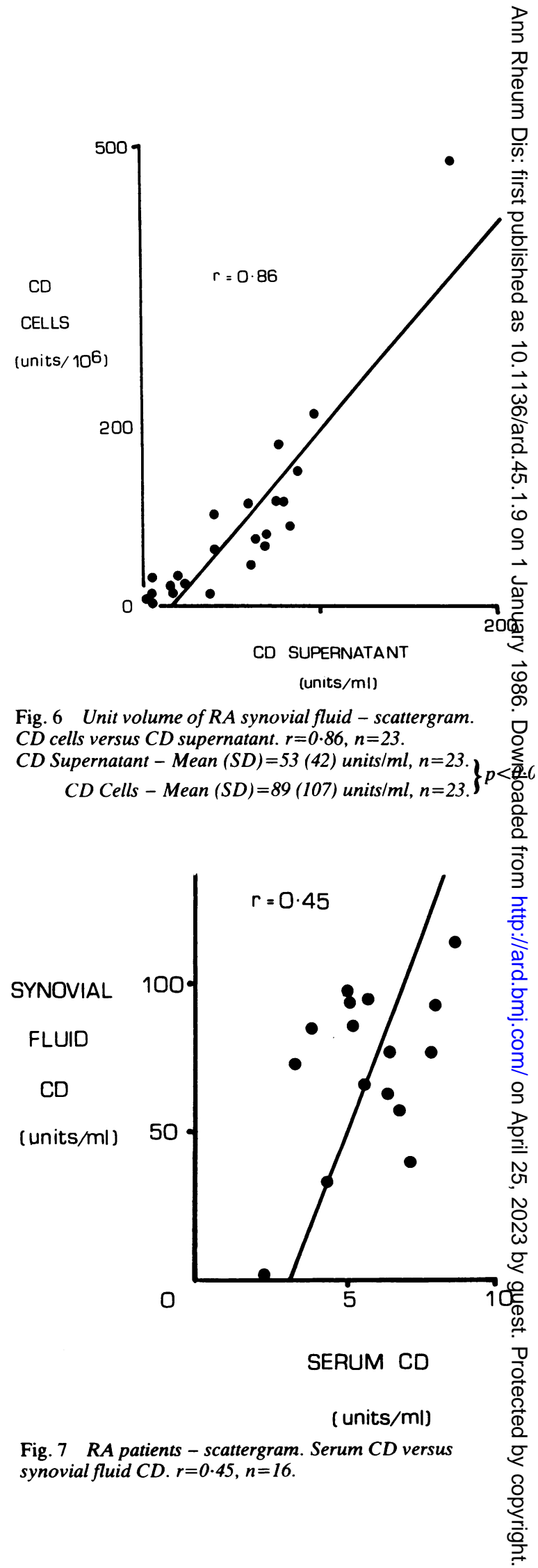


the normal range $(9-50 \mathrm{IU} / \mathrm{l}$ and $50-150 \mathrm{mmol} / \mathrm{l}$ respectively).

There was positive correlation between ESR and CRP $(r=0.69, p<0.001)$, ESR and CD $(r=0.45$, $\mathrm{p}<0.01)$, and CRP and CD $(\mathrm{r}=0.49, \mathrm{p}<0.001)$.

\section{Discussion}

These data suggest that CD is released from SF neutrophils and diffuses into the blood. Synovial fluid $C D$ activity reflects the degree of acute inflammation of an individual joint, and we suggest that serum $C D$ activity is an integrated measure of the acute inflammatory component of overall rheumatoid disease activity.

The technique for measuring $\mathrm{CD}$ activity is cheap and reliable. Several thousands of estimations have been performed in one centre and excellent interand intrabatch reproducibility has been shown. ${ }^{1}$ The method is suitable for large numbers of estimations, and a commercial kit is available (P-ET Test, Helena Labs UK Ltd).

The slightly higher mean serum $\mathrm{CD}$ activity for OA patients compared with normal volunteers may represent a real difference or may be accounted for by demographic differences between the groups. Although we did not record the ages of patients or volunteers, the former were generally elderly females, whereas the latter were male students. However, no significant difference has been reported for serum CD activity between male and female normal volunteers or between normal school children and adult volunteers. ${ }^{1}$

The results of short term storage of SF suggest that significant fluctuations of $\mathrm{CD}$ activity do not occur during four hours at room temperature. It seems reasonable to suppose that variations in the time taken between sample aspiration and freezing would be unlikely to cause important errors in the majority of cases.

Serum measurements of $\gamma$-GT and creatinine were made in an attempt to exclude liver or renal disease, as damage to these organs is associated with raised serum CD levels. ${ }^{1}$ As $\gamma$-GT was raised in only one case and all creatinine estimations fell within the normal range, we feel that this source of error has been reasonably excluded. For completeness the results of the patient with raised $\gamma$-GT have been incorporated into all calculations.

The cellular components of RA SF contained higher $\mathrm{CD}$ activity than the corresponding supernatant, but RA synovial CD activity was no greater than that of 15 normal postmortem specimens previously studied (mean (SD) $=45$ (24) units/g). ${ }^{1}$ This suggests a $C D$ concentration gradient running from cells to fluid to synovium. Neutrophils are the predominant cell type in inflammatory SF. ${ }^{5}$ It has been shown that their half life is about four hours and estimated that in an inflamed rheumatoid knee the daily neutrophil breakdown in the synovial cavity might well exceed one billion cells. ${ }^{6} \mathrm{Neu}-$ trophils contain 20 times more $C D$ than lymphocytes (Fig. 1), so it seems likely that CD originates from SF neutrophils.

As the SF neutrophil count has long been considered the marker of acute inflammation, ${ }^{5}$ the close correlation between it and CD levels $(r=0.93)$ implies that SF CD activity is an accurate measure of the acute inflammatory activity of a joint.

Synovial permeability can be assessed by the ratio of SF/serum concentrations of molecules that are not produced or destroyed in joints. Inflammation increases synovial permeability, so that the ratio of $\mathrm{SF} / \mathrm{serum}$ for transferrin, a protein manufactured solely by the liver and of similar molecular weight to $\mathrm{CD}$, varied from 0.56 in OA patients to 0.84 in RA patients in one study. ${ }^{7}$ Our value of 0.6 for the $C D$ $\mathrm{SF} /$ serum ratio of OA patients agrees well with this, suggesting diffusion of $C D$ from blood to $S F$. In contrast, the $S F /$ serum $C D$ ratios for RA patients $($ mean $=13 \cdot 1)$ reflect the high levels of $C D$ activity in SF compared with serum and suggest that $C D$ originates from inflamed joints and diffuses into the blood.

Such diffusion is reflected by the correlation found between CD activity in serum and SF (Fig. 7). Close correlation would not be expected because of the varying contributions from other joints in different patients.

If it is assumed that all synovial joints react to inflammation in a similar manner and that background $\mathrm{CD}$ production, metabolism, and excretion occur at constant rates, then serum $C D$ activity will be directly proportional to total neutrophil turnover.

Serum CD levels correlated weakly but significantly with ESR $(r=0.45)$ and rather better with CRP $(r=0 \cdot 49)$. The relationship is, however, not so strong as to suggest that $\mathrm{CD}$ estimation is merely a further reflection of the acute phase response but close enough to support the hypothesis that serum $\mathrm{CD}$ activity is a specific measurement of inflammation.

Thus serum $\mathrm{CD}$ estimation may offer an integrated measure of acute inflammation in rheumatoid arthritis. Such a laboratory measure would reflect an entirely different dimension of rheumatoid disease activity compared with indexes such as the ESR.

We thank Drs Barnes, Perry, and Rudge for permission to study patients under their care and Dr Kirwan for help with the manuscript. 


\section{References}

1 Jones D D, Roberts E L. Enzymes of DNA Metabolism in clinical diagnosis. Cambridge: Chancery Press, 1984.

2 Steuart C D, Burke P J. Cytidine deaminase and the development of resistance to arabinosyl cytosine. Nature New Biol 1971; 233: 109-10.

3 Chabot G G, Bouchard J, Momparler R L. Kinetics of deamination of 5-aza-2'-deoxycytidine and cytosine arabinoside by human liver cytidine deaminase and its inhibition by 3 deazauridine, thymidine or uracil arabinoside. Biochem Pharmacol 1983; 32: 1327-8.
4 Jones D D. Bahijri S. Roberts E L. Williams F G. Activity of serum cytidine deaminase during pregnancy. $\mathrm{Br} J$ Obstet Gynaecol 1982; 89: 314-7.

5 Ropes M W. Bauer W. Synovial fluid changes in joint diseases Cambridge. Massachusetts: Harvard University Press, 1953.

6 Hollingsworth J W. Siegel E R. Creasey W A. Granulocyte survival in synovial exudate of patients with rheumatoido arthritis and other inflammatory joint discases. Yale J Biol Med $\vec{D}$ 1967: 39: 289-96.

7 Kushner I. Somerville J A. Permeability of human synovialể membrane to plasma proteins: relationship to molecular size $\rightarrow$ and inflammation. Arthritis Rheum 1971: 14: 56(1-70. 